\title{
SEQUENCES FOR COMPLEXES II
}

\author{
LARS WINTHER CHRISTENSEN
}

\section{Introduction and Notation}

This short paper elaborates on an example given in [4] to illustrate an application of sequences for complexes:

Let $R$ be a local ring with a dualizing complex $D$, and let $M$ be a finitely generated $R$-module; then a sequence $x_{1}, \ldots, x_{n}$ is part of a system of parameters for $M$ if and only if it is a $\mathbf{R H o m}_{R}(M, D)$-sequence $[4,5.10]$.

The final Theorem 3.9 of this paper generalizes the result above in two directions: the dualizing complex is replaced by a Cohen-Macaulay semidualizing complex (see [3, Sec. 2] or 3.8 below for definitions), and the finite module is replaced by a complex with finite homology.

Before we can even state, let alone prove, this generalization of $[4,5.10]$ we have to introduce and study parameters for complexes. For a finite $R$ module $M$ every $M$-sequence is part of a system of parameters for $M$, so, loosely speaking, regular elements are just special parameters. For a complex $X$, however, parameters and regular elements are two different things, and kinship between them implies strong relations between two measures of the size of $X$ : the amplitude and the Cohen-Macaulay defect (both defined below). This is described in 3.5, 3.6, and 3.7.

The definition of parameters for complexes is based on a notion of anchor prime ideals. These do for complexes what minimal prime ideals do for modules, and the quantitative relations between dimension and depth under dagger duality —studied in [3] — have a qualitative description in terms of anchor and associated prime ideals.

Throughout $R$ denotes a commutative, Noetherian local ring with maximal ideal $\mathfrak{m}$ and residue field $k=R / \mathfrak{m}$. We use the same notation as in [4], but for convenience we recall a few basic facts.

The homological position and size of a complex $X$ is captured by the $s u$ -

Received May 2, 2000. 
premum, infimum, and amplitude:

$$
\begin{aligned}
\sup X & =\sup \left\{\ell \in Z \mid \mathrm{H}_{\ell}(X) \neq 0\right\}, \\
\inf X & =\inf \left\{\ell \in Z \mid \mathrm{H}_{\ell}(X) \neq 0\right\}, \quad \text { and } \\
\operatorname{amp} X & =\sup X-\inf X .
\end{aligned}
$$

By convention, $\sup X=-\infty$ and inf $X=\infty$ if $\mathrm{H}(X)=0$.

The support of a complex $X$ is the set

$$
\operatorname{Supp}_{R} X=\left\{\mathfrak{p} \in \operatorname{Spec} R \mid X_{\mathfrak{p}} \nsucceq 0\right\}=\bigcup_{\ell} \operatorname{Supp}_{R} \mathrm{H}_{\ell}(X) .
$$

As usual $\operatorname{Min}_{R} X$ is the subset of minimal elements in the support.

The depth and the (Krull) dimension of an $R$-complex $X$ are defined as follows:

$$
\begin{aligned}
\operatorname{depth}_{R} X & =-\sup \left(\operatorname{RHom}_{R}(k, X)\right), \text { for } X \in \mathscr{D}_{-}(R), \quad \text { and } \\
\operatorname{dim}_{R} X & =\sup \left\{\operatorname{dim} R / \mathfrak{p}-\inf X_{\mathfrak{p}} \mid \mathfrak{p} \in \operatorname{Supp}_{R} X\right\},
\end{aligned}
$$

cf. [6, Sec. 3]. For modules these notions agree with the usual ones. It follows from the definition that

$$
\operatorname{dim}_{R} X \geq \operatorname{dim}_{R_{\mathfrak{p}}} X_{\mathfrak{p}}+\operatorname{dim} R / \mathfrak{p}
$$

for $X \in \mathscr{D}(R)$ and $\mathfrak{p} \in \operatorname{Spec} R$; and there are always inequalities:

$$
\begin{aligned}
& -\inf X \leq \operatorname{dim}_{R} X \quad \text { for } \quad X \in \mathscr{D}_{+}(R) ; \quad \text { and } \\
& -\sup X \leq \operatorname{depth}_{R} X \quad \text { for } \quad X \in \mathscr{D}_{-}(R) \text {. }
\end{aligned}
$$

A complex $X \in \mathscr{D}_{\mathrm{b}}^{\mathrm{f}}(R)$ is Cohen-Macaulay if and only if $\operatorname{dim}_{R} X=$ $\operatorname{depth}_{R} X$, that is, if an only if the Cohen-Macaulay defect,

$$
\operatorname{cmd}_{R} X=\operatorname{dim}_{R} X-\operatorname{depth}_{R} X,
$$

is zero. For complexes in $\mathscr{D}_{\mathrm{b}}^{\mathrm{f}}(R)$ the Cohen-Macaulay defect is always nonnegative, cf. [6, Cor. 3.9].

\section{Anchor Prime Ideals}

In [4] we introduced associated prime ideals for complexes. The analysis of the support of a complex is continued in this section, and the aim is now to identify the prime ideals that do for complexes what the minimal ones do for modules. 
Definitions 2.1. Let $X \in \mathscr{D}_{+}(R)$; we say that $\mathfrak{p} \in \operatorname{Spec} R$ is an anchor prime ideal for $X$ if and only if $\operatorname{dim}_{R_{\mathfrak{p}}} X_{\mathfrak{p}}=-\inf X_{\mathfrak{p}}>-\infty$. The set of anchor prime ideals for $X$ is denoted by $\operatorname{Anc}_{R} X$; that is,

$$
\operatorname{Anc}_{R} X=\left\{\mathfrak{p} \in \operatorname{Supp}_{R} X \mid \operatorname{dim}_{R_{\mathfrak{p}}} X_{\mathfrak{p}}+\inf X_{\mathfrak{p}}=0\right\} .
$$

For $n \in \mathrm{N}_{0}$ we set

$$
\mathrm{W}_{n}(X)=\left\{\mathfrak{p} \in \operatorname{Supp}_{R} X \mid \operatorname{dim}_{R} X-\operatorname{dim} R / \mathfrak{p}+\inf X_{\mathfrak{p}} \leq n\right\} .
$$

ObSERVATION 2.2. Let $S$ be a multiplicative system in $R$, and let $\mathfrak{p} \in$ Spec $R$. If $\mathfrak{p} \cap S=\emptyset$ then $S^{-1} \mathfrak{p}$ is a prime ideal in $S^{-1} R$, and for $X \in$ $\mathscr{D}(R)$ there is an isomorphism $S^{-1} X_{S^{-1} \mathfrak{p}} \simeq X_{\mathfrak{p}}$ in $\mathscr{D}\left(R_{\mathfrak{p}}\right)$. In particular, inf $S^{-1} X_{S^{-1} \mathfrak{p}}=\inf X_{\mathfrak{p}}$ and $\operatorname{dim}_{S^{-1} R_{S^{-1}}} S^{-1} X_{S^{-1} \mathfrak{p}}=\operatorname{dim}_{R_{\mathfrak{p}}} X_{\mathfrak{p}}$. Thus, the next biconditional holds for $X \in \mathscr{D}_{+}(R)$ and $\mathfrak{p} \in \operatorname{Spec} R$ with $\mathfrak{p} \cap S=\emptyset$.

$$
\mathfrak{p} \in \operatorname{Anc}_{R} X \Longleftrightarrow S^{-1} \mathfrak{p} \in \operatorname{Anc}_{S^{-1} R} S^{-1} X .
$$

THeOREM 2.3. For $X \in \mathscr{D}_{+}(R)$ there are inclusions:

$$
\begin{aligned}
\operatorname{Min}_{R} X \subseteq \operatorname{Anc}_{R} X ; \quad \text { and } \\
\mathrm{W}_{0}(X) \subseteq \operatorname{Anc}_{R} X .
\end{aligned}
$$

Furthermore, if $\operatorname{amp} X=0$, that is, if $X$ is equivalent to a module up to a shift, then

$$
\operatorname{Anc}_{R} X=\operatorname{Min}_{R} X \subseteq \operatorname{Ass}_{R} X
$$

and if $X$ is Cohen-Macaulay, that is, $X \in \mathscr{D}_{\mathrm{b}}^{\mathrm{f}}(R)$ and $\operatorname{dim}_{R} X=\operatorname{depth}_{R} X$, then

$$
\operatorname{Ass}_{R} X \subseteq \operatorname{Anc}_{R} X=\mathrm{W}_{0}(X) .
$$

Proof. In the following $X$ belongs to $\mathscr{D}_{+}(R)$.

(a): If $\mathfrak{p}$ belongs to $\operatorname{Min}_{R} X$ then $\operatorname{Supp}_{R_{\mathfrak{p}}} X_{\mathfrak{p}}=\left\{\mathfrak{p}_{\mathfrak{p}}\right\}$, so $\operatorname{dim}_{R_{\mathfrak{p}}} X_{\mathfrak{p}}=$ $-\inf X_{\mathfrak{p}}$, that is, $\mathfrak{p}_{\mathfrak{p}} \in \operatorname{Anc}_{R_{\mathfrak{p}}} X_{\mathfrak{p}}$ and hence $\mathfrak{p} \in \operatorname{Anc}_{R} X$ by (2.1).

(b): Assume that $\mathfrak{p}$ belongs to $\mathrm{W}_{0}(X)$, then $\operatorname{dim}_{R} X=\operatorname{dim} R / \mathfrak{p}-\inf X_{\mathfrak{p}}$, and since $\operatorname{dim}_{R} X \geq \operatorname{dim}_{R_{\mathfrak{p}}} X_{\mathfrak{p}}+\operatorname{dim} R / \mathfrak{p}$ and $\operatorname{dim}_{R_{\mathfrak{p}}} X_{\mathfrak{p}} \geq-\inf X_{\mathfrak{p}}$, cf. (1.1) and (1.2), it follows that $\operatorname{dim}_{R_{\mathfrak{p}}} X_{\mathfrak{p}}=-\inf X_{\mathfrak{p}}$, as desired.

(c): For $M \in \mathscr{D}_{0}(R)$ we have

$$
\operatorname{Anc}_{R} M=\left\{\mathfrak{p} \in \operatorname{Supp}_{R} M \mid \operatorname{dim}_{R_{\mathfrak{p}}} M_{\mathfrak{p}}=0\right\}=\operatorname{Min}_{R} M,
$$


and the inclusion $\operatorname{Min}_{R} M \subseteq \operatorname{Ass}_{R} M$ is well-known.

(d): Assume that $X \in \mathscr{D}_{\mathrm{b}}^{\mathrm{f}}(R)$ and $\operatorname{dim}_{R} X=\operatorname{depth}_{R} X$, then $\operatorname{dim}_{R_{\mathfrak{p}}} X_{\mathfrak{p}}=$ $\operatorname{depth}_{R_{\mathfrak{p}}} X_{\mathfrak{p}}$ for all $\mathfrak{p} \in \operatorname{Supp}_{R} X$, cf. [5, (16.17)]. If $\mathfrak{p} \in \operatorname{Ass}_{R} X$ we have

$$
\operatorname{dim}_{R_{\mathfrak{p}}} X_{\mathfrak{p}}=\operatorname{depth}_{R_{\mathfrak{p}}} X_{\mathfrak{p}}=-\sup X_{\mathfrak{p}} \leq-\inf X_{\mathfrak{p}},
$$

cf. [4, Def. 2.3], and it follows by (1.2) that equality must hold, so $\mathfrak{p}$ belongs to $\mathrm{Anc}_{R} X$.

For each $\mathfrak{p} \in \operatorname{Supp}_{R} X$ there is an equality

$$
\operatorname{dim}_{R} X=\operatorname{dim}_{R_{\mathfrak{p}}} X_{\mathfrak{p}}+\operatorname{dim} R / \mathfrak{p},
$$

cf. [5, (17.4)(b)], so $\operatorname{dim}_{R} X-\operatorname{dim} R / \mathfrak{p}+\inf X_{\mathfrak{p}}=0$ for $\mathfrak{p}$ with $\operatorname{dim}_{R_{\mathfrak{p}}} X_{\mathfrak{p}}=$ $-\inf X_{\mathfrak{p}}$. This proves the inclusion $\operatorname{Anc}_{R} X \subseteq \mathrm{W}_{0}(X)$.

Corollary 2.4. For $X \in \mathscr{D}_{\mathrm{b}}(R)$ there is an inclusion:

$$
\operatorname{Min}_{R} X \subseteq \operatorname{Ass}_{R} X \cap \operatorname{Anc}_{R} X
$$

and for $\mathfrak{p} \in \operatorname{Ass}_{R} X \cap \operatorname{Anc}_{R} X$ there is an equality:

$$
\operatorname{cmd}_{R_{\mathfrak{p}}} X_{\mathfrak{p}}=\operatorname{amp} X_{\mathfrak{p}}
$$

Proof. Part (a) follows by 2.3 (a) and [4, Prop. 2.6]; part (b) is immediate by the definitions of associated and anchor prime ideals, cf. [4, Def. 2.3].

Corollary 2.5. If $X \in \mathscr{D}_{+}^{\mathrm{f}}(R)$, then

$$
\operatorname{dim}_{R} X=\sup \left\{\operatorname{dim} R / \mathfrak{p}+\operatorname{dim}_{R_{\mathfrak{p}}} X_{\mathfrak{p}} \mid \mathfrak{p} \in \operatorname{Anc}_{R} X\right\} .
$$

Proof. It is immediate by the definitions that

$$
\begin{aligned}
\operatorname{dim}_{R} X & =\sup \left\{\operatorname{dim} R / \mathfrak{p}-\inf X_{\mathfrak{p}} \mid \mathfrak{p} \in \operatorname{Supp}_{R} X\right\} \\
& \geq \sup \left\{\operatorname{dim} R / \mathfrak{p}-\inf X_{\mathfrak{p}} \mid \mathfrak{p} \in \operatorname{Anc}_{R} X\right\} \\
& =\sup \left\{\operatorname{dim} R / \mathfrak{p}+\operatorname{dim}_{R_{\mathfrak{p}}} X_{\mathfrak{p}} \mid \mathfrak{p} \in \operatorname{Anc}_{R} X\right\} ;
\end{aligned}
$$

and the opposite inequality follows by 2.3 (b).

Proposition 2.6. The following hold:

(a) If $X \in \mathscr{D}_{+}(R)$ and $\mathfrak{p}$ belongs to $\operatorname{Anc}_{R} X$, then $\operatorname{dim}_{R_{\mathfrak{p}}}\left(\mathrm{H}_{\mathrm{inf} X_{\mathfrak{p}}}\left(X_{\mathfrak{p}}\right)\right)=0$.

(b) If $X \in \mathscr{D}_{\mathrm{b}}^{\mathrm{f}}(R)$, then $\mathrm{Anc}_{R} X$ is a finite set.

Proof. (a): Assume that $\mathfrak{p} \in \mathrm{Anc}_{R} X$; by [6, Prop. 3.5] we have

$$
-\inf X_{\mathfrak{p}}=\operatorname{dim}_{R_{\mathfrak{p}}} X_{\mathfrak{p}} \geq \operatorname{dim}_{R_{\mathfrak{p}}}\left(\mathrm{H}_{\text {inf } X_{\mathfrak{p}}}\left(X_{\mathfrak{p}}\right)\right)-\inf X_{\mathfrak{p}},
$$


and hence $\operatorname{dim}_{R_{\mathfrak{p}}}\left(\mathrm{H}_{\text {inf } X_{\mathfrak{p}}}\left(X_{\mathfrak{p}}\right)\right)=0$.

(b): By (a) every anchor prime ideal for $X$ is minimal for one of the homology modules of $X$, and when $X \in \mathscr{D}_{\mathrm{b}}^{\mathrm{f}}(R)$ each of the finitely many homology modules has a finite number of minimal prime ideals.

ObServation 2.7. By Nakayama's lemma it follows that

$$
\inf \mathrm{K}\left(x_{1}, \ldots, x_{n} ; Y\right)=\inf Y,
$$

for $Y \in \mathscr{D}_{+}^{\mathrm{f}}(R)$ and elements $x_{1}, \ldots, x_{n} \in \mathfrak{m}$.

Proposition 2.8 (Dimension of Koszul Complexes). The following hold for a complex $Y \in \mathscr{D}_{+}^{\mathrm{f}}(R)$ and elements $x_{1}, \ldots, x_{n} \in \mathfrak{m}$ :

(a) $\operatorname{dim}_{R} \mathrm{~K}\left(x_{1}, \ldots, x_{n} ; Y\right)$

$$
=\sup \left\{\operatorname{dim} R / \mathfrak{p}-\inf Y_{\mathfrak{p}} \mid \mathfrak{p} \in \operatorname{Supp}_{R} Y \cap \mathrm{V}\left(x_{1}, \ldots, x_{n}\right)\right\} ; \quad \text { and }
$$

(b) $\operatorname{dim}_{R} Y-n \leq \operatorname{dim}_{R} \mathrm{~K}\left(x_{1}, \ldots, x_{n} ; Y\right) \leq \operatorname{dim}_{R} Y$.

Furthermore:

(c) The elements $x_{1}, \ldots, x_{n}$ are contained in a prime ideal $\mathfrak{p} \in \mathrm{W}_{n}(Y) ;$ and

(d) $\operatorname{dim}_{R} \mathrm{~K}\left(x_{1}, \ldots, x_{n} ; Y\right)=\operatorname{dim}_{R} Y$ if and only if $x_{1}, \ldots, x_{n} \in \mathfrak{p}$ for some $\mathfrak{p} \in \mathrm{W}_{0}(Y)$.

Proof. Since $\operatorname{Supp}_{R} \mathrm{~K}\left(x_{1}, \ldots, x_{n} ; Y\right)=\operatorname{Supp}_{R} Y \cap \mathrm{V}\left(x_{1}, \ldots, x_{n}\right)$ (see [6, p. 157] and [4, 3.2]) (a) follows by the definition of Krull dimension and 2.7. In (b) the second inequality follows from (a); the first one is established through four steps:

$1^{\circ} Y=R$ : The second equality below follows from the definition of Krull dimension as $\operatorname{Supp}_{R} \mathrm{~K}\left(x_{1}, \ldots, x_{n}\right)=\operatorname{Supp}_{R} \mathrm{H}_{0}\left(\mathrm{~K}\left(x_{1}, \ldots, x_{n}\right)\right)=$ $\mathrm{V}\left(x_{1}, \ldots, x_{n}\right)$, cf. $[4,3.2]$; the inequality is a consequence of Krull's Principal Ideal Theorem, see for example [8, Thm. 13.6].

$$
\begin{aligned}
\operatorname{dim}_{R} \mathrm{~K}\left(x_{1}, \ldots, x_{n} ; Y\right) & =\operatorname{dim}_{R} \mathrm{~K}\left(x_{1}, \ldots, x_{n}\right) \\
& =\sup \left\{\operatorname{dim} R / \mathfrak{p} \mid \mathfrak{p} \in \mathrm{V}\left(x_{1}, \ldots, x_{n}\right)\right\} \\
& =\operatorname{dim} R /\left(x_{1}, \ldots, x_{n}\right) \\
& \geq \operatorname{dim} R-n \\
& =\operatorname{dim}_{R} Y-n .
\end{aligned}
$$


$2^{\circ} Y=B$, a cyclic module: By $\bar{x}_{1}, \ldots, \bar{x}_{n}$ we denote the residue classes in $B$ of the elements $x_{1}, \ldots, x_{n}$; the inequality below is by $1^{\circ}$.

$$
\begin{aligned}
\operatorname{dim}_{R} \mathrm{~K}\left(x_{1}, \ldots, x_{n} ; Y\right) & =\operatorname{dim}_{R} \mathrm{~K}\left(\bar{x}_{1}, \ldots, \bar{x}_{n}\right) \\
& =\operatorname{dim}_{B} \mathrm{~K}\left(\bar{x}_{1}, \ldots, \bar{x}_{n}\right) \\
& \geq \operatorname{dim} B-n \\
& =\operatorname{dim}_{R} Y-n .
\end{aligned}
$$

$3^{\circ} Y=H \in \mathscr{D}_{0}^{\mathrm{f}}(R)$ : We set $B=R / \operatorname{Ann}_{R} H$; the first equality below follows by [6, Prop. 3.11] and the inequality by $2^{\circ}$.

$$
\begin{aligned}
\operatorname{dim}_{R} \mathrm{~K}\left(x_{1}, \ldots, x_{n} ; Y\right) & =\operatorname{dim}_{R} \mathrm{~K}\left(x_{1}, \ldots, x_{n} ; B\right) \\
& \geq \operatorname{dim}_{R} B-n \\
& =\operatorname{dim}_{R} Y-n .
\end{aligned}
$$

$4^{\circ} Y \in \mathscr{D}_{\mathrm{b}}^{\mathrm{f}}(R)$ : The first equality below follows by [6, Prop. 3.12] and the last by [6, Prop. 3.5]; the inequality is by $3^{\circ}$.

$$
\begin{aligned}
\operatorname{dim}_{R} \mathrm{~K}\left(x_{1}, \ldots, x_{n} ; Y\right) & =\sup \left\{\operatorname{dim}_{R} \mathrm{~K}\left(x_{1}, \ldots, x_{n} ; \mathrm{H}_{\ell}(Y)\right)-\ell \mid \ell \in \mathrm{Z}\right\} \\
& \geq \sup \left\{\operatorname{dim}_{R} \mathrm{H}_{\ell}(Y)-n-\ell \mid \ell \in \mathbf{Z}\right\} \\
& =\operatorname{dim}_{R} Y-n .
\end{aligned}
$$

This proves (b).

In view of (a) it now follows that

$$
\operatorname{dim}_{R} Y-n \leq \operatorname{dim} R / \mathfrak{p}-\inf Y_{\mathfrak{p}}
$$

for some $\mathfrak{p} \in \operatorname{Supp}_{R} Y \cap \mathrm{V}\left(x_{1}, \ldots, x_{n}\right)$. That is, the elements $x_{1}, \ldots, x_{n}$ are contained in a prime ideal $\mathfrak{p} \in \operatorname{Supp}_{R} Y$ with

$$
\operatorname{dim}_{R} Y-\operatorname{dim} R / \mathfrak{p}+\inf Y_{\mathfrak{p}} \leq n,
$$

and this proves (c).

Finally, it is immediate by the definitions that

$$
\operatorname{dim}_{R} Y=\sup \left\{\operatorname{dim} R / \mathfrak{p}-\inf Y_{\mathfrak{p}} \mid \mathfrak{p} \in \operatorname{Supp}_{R} Y \cap \mathrm{V}\left(x_{1}, \ldots, x_{n}\right)\right\}
$$

if and only if $\mathrm{W}_{0}(Y) \cap \mathrm{V}\left(x_{1}, \ldots, x_{n}\right) \neq \emptyset$. This proves (d). 
THeOREM 2.9. If $Y \in \mathscr{D}_{\mathrm{b}}^{\mathrm{f}}(R)$, then the next two numbers are equal.

$$
\begin{aligned}
& \mathrm{d}(Y)=\operatorname{dim}_{R} Y+\inf Y ; \quad \text { and } \\
& \mathrm{s}(Y)=\inf \left\{s \in \mathrm{N}_{0} \mid \exists x_{1}, \ldots, x_{s}: \mathfrak{m} \in \operatorname{Anc}_{R} \mathrm{~K}\left(x_{1}, \ldots, x_{s} ; Y\right)\right\} .
\end{aligned}
$$

Proof. There are two inequalities to prove.

$\mathrm{d}(Y) \leq \mathrm{s}(Y)$ : Let $x_{1}, \ldots, x_{s} \in \mathfrak{m}$ be such that $\mathfrak{m} \in \operatorname{Anc}_{R} \mathrm{~K}\left(x_{1}, \ldots, x_{s} ; Y\right)$; by 2.8 (b) and 2.7 we then have

$$
\operatorname{dim}_{R} Y-s \leq \operatorname{dim}_{R} \mathrm{~K}\left(x_{1}, \ldots, x_{s} ; Y\right)=-\inf \mathrm{K}\left(x_{1}, \ldots, x_{s} ; Y\right)=-\inf Y,
$$

so $\mathrm{d}(Y) \leq s$, and the desired inequality follows.

$\mathrm{s}(Y) \leq \mathrm{d}(Y)$ : We proceed by induction on $\mathrm{d}(\mathrm{Y})$. If $\mathrm{d}(Y)=0$ then $\mathfrak{m} \in$ $\operatorname{Anc}_{R} Y$ so s $(Y)=0$. If $\mathrm{d}(Y)>0$ then $\mathfrak{m} \notin \operatorname{Anc}_{R} Y$, and since $\operatorname{Anc}_{R} Y$ is a finite set, by 2.6(b), we can choose an element $x \in \mathfrak{m}-\cup_{\mathfrak{p} \in \operatorname{Anc}_{R} Y} \mathfrak{p}$. We set $K=\mathrm{K}(x ; Y)$; it is cleat that $\mathrm{s}(Y) \leq \mathrm{s}(K)+1$. Furthermore, it follows by 2.8 (a) and 2.3 (b) that $\operatorname{dim}_{R} K<\operatorname{dim}_{R} Y$ and thereby d( $\left.K\right)<\mathrm{d}(Y)$, cf. 2.7 . Thus, by the induction hypothesis we have

$$
\mathrm{s}(Y) \leq \mathrm{s}(K)+1 \leq \mathrm{d}(K)+1 \leq \mathrm{d}(Y)
$$

as desired.

\section{Parameters}

By 2.9 the next definitions extend the classical notions of systems and sequences of parameters for finite modules (e.g., see $[8, \S 14]$ and the appendix in [2]).

Definitions 3.1. Let $Y$ belong to $\mathscr{D}_{\mathrm{b}}^{\mathrm{f}}(R)$ and set $d=\operatorname{dim}_{R} Y+\inf Y$. A set of elements $x_{1}, \ldots, x_{d} \in \mathfrak{m}$ are said to be a system of parameters for $Y$ if and only if $\mathrm{m} \in \mathrm{Anc}_{R} \mathrm{~K}\left(x_{1}, \ldots, x_{d} ; Y\right)$.

A sequence $\boldsymbol{x}=x_{1}, \ldots, x_{n}$ is said to be a $Y$-parameter sequence if and only if it is part of a system of parameters for $Y$.

Lemma 3.2. Let $Y$ belong to $\mathscr{D}_{\mathrm{b}}^{\mathrm{f}}(R)$ and set $d=\operatorname{dim}_{R} Y+\inf Y$. The next two conditions are equivalent for elements $x_{1}, \ldots, x_{d} \in \mathfrak{m}$.

(i) $x_{1}, \ldots, x_{d}$ is a system of parameters for $Y$.

(ii) For every $j \in\{0, \ldots, d\}$ there is an equality:

$$
\operatorname{dim}_{R} \mathrm{~K}\left(x_{1}, \ldots, x_{j} ; Y\right)=\operatorname{dim}_{R} Y-j ;
$$

and $x_{j+1}, \ldots, x_{d}$ is a system of parameters for $\mathrm{K}\left(x_{1}, \ldots, x_{j} ; Y\right)$. 
Proof. (i) $\Rightarrow$ (ii): Assume that $x_{1}, \ldots, x_{d}$ is a system of parameters for $Y$, then

$$
\begin{array}{rlr}
-\inf \mathrm{K}\left(x_{1}, \ldots, x_{d} ; Y\right) & =\operatorname{dim}_{R} \mathrm{~K}\left(x_{1}, \ldots, x_{d} ; Y\right) \\
& =\operatorname{dim}_{R} \mathrm{~K}\left(x_{j+1}, \ldots, x_{d} ; \mathrm{K}\left(x_{1}, \ldots, x_{j} ; Y\right)\right. \\
& \geq \operatorname{dim}_{R} \mathrm{~K}\left(x_{1}, \ldots, x_{j} ; Y\right)-(d-j) & \text { by } 2.8(\mathrm{~b}) \\
& \geq \operatorname{dim}_{R} Y-j-(d-j) & \text { by } 2.8(\mathrm{~b}) \\
& =\operatorname{dim}_{R} Y-d \\
& =-\inf Y .
\end{array}
$$

By 2.7 it now follows that $-\inf Y=\operatorname{dim}_{R} \mathrm{~K}\left(x_{1}, \ldots, x_{j} ; Y\right)-(d-j)$, so

$$
\operatorname{dim}_{R} \mathrm{~K}\left(x_{1}, \ldots, x_{j} ; Y\right)=d-j-\inf Y=\operatorname{dim}_{R} Y-j,
$$

as desired. It also follows that $\mathrm{d}\left(\mathrm{K}\left(x_{1}, \ldots, x_{j} ; Y\right)\right)=d-j$, and since

$$
\mathfrak{m} \in \mathrm{Anc}_{R} \mathrm{~K}\left(x_{1}, \ldots, x_{d} ; Y\right)=\operatorname{Anc}_{R} \mathrm{~K}\left(x_{j+1}, \ldots, x_{d} ; \mathrm{K}\left(x_{1}, \ldots, x_{j} ; Y\right)\right) \text {, }
$$

we conclude that $x_{j+1}, \ldots, x_{d}$ is a system of parameters for $\mathrm{K}\left(x_{1}, \ldots, x_{j} ; Y\right)$.

(ii) $\Rightarrow$ (i): If $\operatorname{dim}_{R} \mathrm{~K}\left(x_{1}, \ldots, x_{j} ; Y\right)=\operatorname{dim}_{R} Y-j$ then $\mathrm{d}\left(\mathrm{K}\left(x_{1}, \ldots, x_{j} ; Y\right)\right)$ $=d-j$; and if $x_{j+1}, \ldots, x_{d}$ is a system of parameters for $\mathrm{K}\left(x_{1}, \ldots, x_{j} ; Y\right)$ then $\mathfrak{m}$ belongs to

$$
\operatorname{Anc}_{R} \mathrm{~K}\left(x_{j+1}, \ldots, x_{d} ; \mathrm{K}\left(x_{1}, \ldots, x_{j} ; Y\right)\right)=\operatorname{Anc}_{R} \mathrm{~K}\left(x_{1}, \ldots, x_{d} ; Y\right) \text {, }
$$

so $x_{1}, \ldots, x_{d}$ must be a system of parameters for $Y$.

Proposition 3.3. Let $Y \in \mathscr{D}_{\mathrm{b}}^{\mathrm{f}}(R)$. The following conditions are equivalent for a sequence $\boldsymbol{x}=x_{1}, \ldots, x_{n}$ in $\mathrm{m}$.

(i) $\boldsymbol{x}$ is a $Y$-parameter sequence.

(ii) For each $j \in\{0, \ldots, n\}$ there is an equality:

$$
\operatorname{dim}_{R} \mathrm{~K}\left(x_{1}, \ldots, x_{j} ; Y\right)=\operatorname{dim}_{R} Y-j
$$

and $x_{j+1}, \ldots, x_{n}$ is a $\mathrm{K}\left(x_{1}, \ldots, x_{j} ; Y\right)$-parameter sequence.

(iii) There is an equality:

$$
\operatorname{dim}_{R} \mathrm{~K}\left(x_{1}, \ldots, x_{n} ; Y\right)=\operatorname{dim}_{R} Y-n .
$$

Proof. It follows by 3.2 that (i) implies (ii), and (iii) follows from (ii). Now, set $K=\mathrm{K}(\boldsymbol{x} ; Y)$ and assume that $\operatorname{dim}_{R} K=\operatorname{dim}_{R} Y-n$. Choose, by 2.9, 
$s=\mathrm{s}(K)=\operatorname{dim}_{R} K+\inf K$ elements $w_{1}, \ldots, w_{s}$ in $\mathfrak{m}$ such that $\mathfrak{m}$ belongs to $\mathrm{Anc}_{R} \mathrm{~K}\left(w_{1}, \ldots, w_{s} ; K\right)=\mathrm{Anc}_{R} \mathrm{~K}\left(x_{1}, \ldots, x_{n}, w_{1}, \ldots, w_{s} ; Y\right)$. Then, by 2.7, we have

$$
n+s=\left(\operatorname{dim}_{R} Y-\operatorname{dim}_{R} K\right)+\left(\operatorname{dim}_{R} K+\inf K\right)=\operatorname{dim}_{R} Y+\inf Y=d,
$$

so $x_{1}, \ldots, x_{n}, w_{1}, \ldots, w_{s}$ is a system of parameters for $Y$, whence $x_{1}, \ldots, x_{n}$ is a $Y$-parameter sequence.

We now recover a classical result (e.g., see [2, Prop. A.4]):

CoROllary 3.4. Let $M$ be an R-module. The following conditions are equivalent for a sequence $\boldsymbol{x}=x_{1}, \ldots, x_{n}$ in $\mathrm{m}$.

(i) $\boldsymbol{x}$ is an M-parameter sequence.

(ii) For each $j \in\{0, \ldots, n\}$ there is an equality:

$$
\operatorname{dim}_{R} M /\left(x_{1}, \ldots, x_{j}\right) M=\operatorname{dim}_{R} M-j ;
$$

and $x_{j+1}, \ldots, x_{n}$ is an $M /\left(x_{1}, \ldots, x_{j}\right) M$-parameter sequence.

(iii) There is an equality:

$$
\operatorname{dim}_{R} M /\left(x_{1}, \ldots, x_{n}\right) M=\operatorname{dim}_{R} M-n .
$$

Proof. By [6, Prop. 3.12] and [5, (16.22)] we have

$$
\begin{array}{rl}
\operatorname{dim}_{R} & \mathrm{~K}\left(x_{1}, \ldots, x_{j} ; M\right) \\
= & \sup \left\{\operatorname{dim}_{R}\left(M \otimes_{R}^{\mathbf{L}} \mathrm{H}_{\ell}\left(\mathrm{K}\left(x_{1}, \ldots, x_{j}\right)\right)\right)-\ell \mid \ell \in \mathrm{Z}\right\} \\
& =\sup \left\{\operatorname{dim}_{R}\left(M \otimes_{R} \mathrm{H}_{\ell}\left(\mathrm{K}\left(x_{1}, \ldots, x_{j}\right)\right)\right)-\ell \mid \ell \in \mathbf{Z}\right\} \\
& =\operatorname{dim}_{R}\left(M \otimes_{R} R /\left(x_{1}, \ldots, x_{j}\right)\right) .
\end{array}
$$

TheORem 3.5. Let $Y \in \mathscr{D}_{\mathrm{b}}^{\mathrm{f}}(R)$. The following hold for a sequence $\boldsymbol{x}=$ $x_{1}, \ldots, x_{n}$ in $\mathrm{m}$.

(a) There is an inequality:

$$
\operatorname{amp} \mathrm{K}(\boldsymbol{x} ; Y) \geq \operatorname{amp} Y ;
$$

and equality holds if and only if $\boldsymbol{x}$ is a $Y$-sequence.

(b) There is an inequality:

$$
\operatorname{cmd}_{R} \mathrm{~K}(\boldsymbol{x} ; Y) \geq \operatorname{cmd}_{R} Y ;
$$

and equality holds if and only if $\boldsymbol{x}$ is a $Y$-parameter sequence. 
(c) If $\boldsymbol{x}$ is a maximal $Y$-sequence, then

$$
\operatorname{amp} Y \leq \operatorname{cmd}_{R} \mathrm{~K}(\boldsymbol{x} ; Y) .
$$

(d) If $\boldsymbol{x}$ is a system of parameters for $Y$, then

$$
\operatorname{cmd}_{R} Y \leq \operatorname{ampK}(\boldsymbol{x} ; Y) .
$$

Proof. In the following $K$ denotes the Koszul complex $\mathrm{K}(\boldsymbol{x} ; Y)$.

(a): Immediate by 2.7 and [4, Prop. 5.1].

(b): By [4, Thm. 4.7 (a)] and 2.8 (b) we have

$\operatorname{cmd}_{R} K=\operatorname{dim}_{R} K-\operatorname{depth}_{R} K=\operatorname{dim}_{R} K+n-\operatorname{depth}_{R} Y \geq \operatorname{cmd}_{R} Y$,

and by 3.3 equality holds if and only if $\boldsymbol{x}$ is a $Y$-parameter sequence.

(c): Suppose $\boldsymbol{x}$ is a maximal $Y$-sequence, then

$$
\begin{aligned}
\operatorname{amp} Y & =\sup Y-\inf K & & \text { by } 2.7 \\
& =-\operatorname{depth}_{R} K-\inf K & & \text { by [4, Thm. 5.4] } \\
& \leq \operatorname{cmd}_{R} K & & \text { by (1.2). }
\end{aligned}
$$

(d): Suppose $\boldsymbol{x}$ is system of parameters for $Y$, then

$$
\begin{aligned}
\operatorname{amp} K & =\sup K+\operatorname{dim}_{R} K & & \\
& \geq \operatorname{dim}_{R} K-\operatorname{depth}_{R} K & & \text { by (1.3) } \\
& =\operatorname{cmd}_{R} Y & & \text { by (b). }
\end{aligned}
$$

THEOREM 3.6. The following hold for $Y \in \mathscr{D}_{\mathrm{b}}^{\mathrm{f}}(R)$.

(a) The next four conditions are equivalent.

(i) There is a maximal $Y$-sequence which is also a $Y$-parameter sequence.

(ii) $\operatorname{depth}_{R} Y+\sup Y \leq \operatorname{dim}_{R} Y+\inf Y$.

(ii') amp $Y \leq \operatorname{cmd}_{R} Y$.

(iii) There is a maximal strong $Y$-sequence which is also a $Y$-parameter sequence.

(b) The next four conditions are equivalent.

(i) There is a system of parameters for $Y$ which is also a $Y$-sequence.

(ii) $\operatorname{dim}_{R} Y+\inf Y \leq \operatorname{depth}_{R} Y+\sup Y$.

(ii') $\operatorname{cmd}_{R} Y \leq \operatorname{amp} Y$. 
(iii) There is a system of parameters for $Y$ which is also a strong $Y$ sequence.

(c) The next four conditions are equivalent.

(i) There is a system of parameters for $Y$ which is also a maximal $Y$ sequence.

(ii) $\operatorname{dim}_{R} Y+\inf Y=\operatorname{depth}_{R} Y+\sup Y$.

(ii') $\operatorname{cmd}_{R} Y=\operatorname{amp} Y$.

(iii) There is a system of parameters for $Y$ which is also a maximal strong $Y$-sequence.

Proof. Let $Y \in \mathscr{D}_{\mathrm{b}}^{\mathrm{f}}(R)$, set $\mathrm{n}(Y)=\operatorname{depth}_{R} Y+\sup Y$ and $\mathrm{d}(Y)=$ $\operatorname{dim}_{R} Y+\inf Y$.

(a): A maximal $Y$-sequence is of length $\mathrm{n}(Y)$, cf. [4, Cor. 5.5], and the length of a $Y$-parameter sequence is at most $\mathrm{d}(Y)$. Thus, (i) implies (ii) which in turn is equivalent to (ii'). Furthermore, a maximal strong $Y$-sequence is, in particular, a maximal $Y$-sequence, cf. [4, Cor. 5.7], so (iii) is stronger than (i). It is now sufficient to prove the implication (ii) $\Rightarrow$ (iii): We proceed by induction. If $\mathrm{n}(Y)=0$ then the empty sequence is a maximal strong $Y$-sequence and a $Y$-parameter sequence. Let $\mathrm{n}(Y)>0$; the two sets $\operatorname{Ass}_{R} Y$ and $\mathrm{W}_{0}(Y)$ are both finite, and since $0<\mathrm{n}(Y) \leq \mathrm{d}(Y)$ none of them contain $\mathfrak{m}$. We can, therefore, choose an element $x \in \mathfrak{m}-\cup_{\mathrm{Ass}_{R} Y \cup \mathrm{W}_{0}(Y)} \mathfrak{p}$, and $x$ is then a strong $Y$-sequence, cf. [4, Def. 3.3], and a $Y$-parameter sequence, cf. 3.3 and 2.8. Set $K=\mathrm{K}(x ; Y)$, by [4, Thm. 4.7 and Prop. 5.1], respectively, 2.8 and 2.7 we have

$$
\operatorname{depth}_{R} K+\sup K=\mathrm{n}(Y)-1 \leq \mathrm{d}(Y)-1=\operatorname{dim}_{R} K+\inf K .
$$

By the induction hypothesis there exists a maximal strong $K$-sequence $w_{1}, \ldots$, $w_{n-1}$ which is also a $K$-parameter sequence, and it follows by [4, 3.5] and 3.3 that $x, w_{1}, \ldots, w_{n-1}$ is a strong $Y$-sequence and a $Y$-parameter sequence, as wanted.

The proof of (b) i similar to the proof of (a), and (c) follows immediately by (a) and (b).

THEOREM 3.7. The following hold for $Y \in \mathscr{D}_{\mathrm{b}}^{\mathrm{f}}(R)$ :

(a) If amp $Y=0$, then any $Y$-sequence is a $Y$-parameter sequence.

(b) If $\mathrm{cmd}_{R} Y=0$, then any $Y$-parameter sequence is a strong $Y$-sequence.

Proof. The empty sequence is a $Y$-parameter sequence as well as a strong $Y$-sequence, this founds the base for a proof by induction on the length $n$ of 
the sequence $\boldsymbol{x}=x_{1}, \ldots, x_{n}$. Let $n>0$ and set $K=\mathrm{K}\left(x_{1}, \ldots, x_{n-1} ; Y\right)$; by 2.8 (a) we have

$(*)$

$$
\begin{aligned}
\operatorname{dim}_{R} \mathrm{~K}\left(x_{1}, \ldots, x_{n} ; Y\right) & =\operatorname{dim}_{R} \mathrm{~K}\left(x_{n} ; K\right) \\
& =\sup \left\{\operatorname{dim} R / \mathfrak{p}-\inf K_{\mathfrak{p}} \mid \mathfrak{p} \in \operatorname{Supp}_{R} K \cap \mathrm{V}\left(x_{n}\right)\right\} .
\end{aligned}
$$

Assume that amp $Y=0$. If $\boldsymbol{x}$ is a $Y$-sequence, then amp $K=0$ by 3.5 (a) and $x_{n} \notin \mathrm{z}_{R} K$, cf. [4, Def. 3.3]. As $\mathrm{z}_{R} K=\cup_{\mathfrak{p} \in \operatorname{Ass}_{R} K} \mathfrak{p}$, cf. [4, 2.5], it follows by (b) and (c) in 2.3 that $x_{n}$ is not contained in any prime ideal $\mathfrak{p} \in \mathrm{W}_{0}(K)$; so from $(*)$ we conclude that $\operatorname{dim}_{R} \mathrm{~K}\left(x_{n} ; K\right)<\operatorname{dim}_{R} K$, and it follows by 2.8 (b) that $\operatorname{dim}_{R} \mathrm{~K}\left(x_{n}, K\right)=\operatorname{dim}_{R} K-1$. By the induction hypothesis $\operatorname{dim}_{R} K=$ $\operatorname{dim}_{R} Y-(n-1)$, so $\operatorname{dim}_{R} \mathrm{~K}\left(x_{1}, \ldots, x_{n} ; Y\right)=\operatorname{dim}_{R} Y-n$ and it follows by 3.3 that $\boldsymbol{x}$ is a $Y$-parameter sequence. This proves (a).

We now assume that $\operatorname{cmd}_{R} Y=0$. If $\boldsymbol{x}$ is a $Y$-parameter sequence then, by the induction hypothesis, $x_{1}, \ldots, x_{n-1}$ is a strong $Y$-sequence, so it is sufficient to prove that $x_{n} \notin \mathrm{Z}_{R} K$, cf. $[4,3.5]$. By 3.3 it follows that $x_{n}$ is a $K$-parameter sequence, so $\operatorname{dim}_{R} \mathrm{~K}\left(x_{n} ; K\right)=\operatorname{dim}_{R} K-1$ and we conclude from (*) that $x_{n} \notin \cup_{\mathfrak{p} \in \mathrm{W}_{0}(K)} \mathfrak{p}$. Now, by 3.5 (b) we have $\mathrm{cmd}_{R} K=0$, so it follows from 2.3 (d) that $x_{n} \notin \cup_{\mathfrak{p} \in \operatorname{Ass}_{R} K} \mathfrak{p}=\mathrm{Z}_{R} K$. This proves (b).

Semi-Dualizing Complexes 3.8. We recall two basic definitions from [3]:

A complex $C \in \mathscr{D}_{\mathrm{b}}^{\mathrm{f}}(R)$ is said to be semi-dualizing for $R$ if and only if the homothety morphism $\chi_{C}^{R}: R \rightarrow \mathbf{R H o m}_{R}(C, C)$ is an isomorphism [3, (2.1)].

Let $C$ be a semi-dualizing complex for $R$. A complex $Y \in \mathscr{D}_{\mathrm{b}}^{\mathrm{f}}(R)$ is said to be $C$-reflexive if and only if the dagger dual $Y^{\dagger c}=\mathbf{R} \operatorname{Hom}_{R}(Y, C)$ belongs to $\mathscr{D}_{\mathrm{b}}^{\mathrm{f}}(R)$ and the biduality morphism $\delta_{Y}^{C}: Y \rightarrow \mathbf{R H o m}_{R}\left(\mathbf{R H o m}_{R}(Y, C), C\right)$ is invertible in $\mathscr{D}(R)$ [3, (2.7)].

Relations between dimension and depth for $C$-reflexive complexes are studied in [3, sec. 3], and the next result is an immediate consequence of [3, (3.1) and (2.10)].

Let $C$ be a semi-dualizing complex for $R$ and let $Z$ be a $C$-reflexive complex. The following holds for $\mathfrak{p} \in \operatorname{Spec} R$ : If $\mathfrak{p} \in \operatorname{Anc}_{R} Z$ then $\mathfrak{p} \in \operatorname{Ass}_{R} Z^{\dagger c}$, and the converse holds in $C$ is Cohen-Macaulay.

A dualizing complex, cf. [7], is a semi-dualizing complex of finite injective dimension, in particular, it is Cohen-Macaulay, cf. [3, (3.5)]. If $D$ is a dualizing complex for $R$, then, by [7, Prop. V.2.1], all complexes $Y \in \mathscr{D}_{\mathrm{b}}^{\mathrm{f}}(R)$ are $D$ reflexive; in particular, all finite $R$-modules are $D$-reflexive and, therefore, [4, 5.10] is a special case of the following: 
Theorem 3.9. Let $C$ be a Cohen-Macaulay semi-dualizing complex for $R$, and let $\boldsymbol{x}=x_{1}, \ldots, x_{n}$ be a sequence in $\mathrm{m}$. If $Y$ is $C$-reflexive, then $\boldsymbol{x}$ is a $Y$-parameter sequence if and only if it is a $\mathbf{R H o m}_{R}(Y, C)$-sequence; that is

$$
\boldsymbol{x} \text { is a } Y \text {-parameter sequence } \Longleftrightarrow \boldsymbol{x} \text { is a } \mathbf{R H o m}_{R}(Y, C) \text {-sequence. }
$$

Proof. We assume that $C$ is a Cohen-Macaulay semi-dualizing complex for $R$ and that $Y$ is $C$-reflexive, cf. 3.8. The desired biconditional follows by the next chain, and each step is explained below (we use the notation $-{ }^{\dagger c}$ introduced in 3.8).

$$
\begin{aligned}
\boldsymbol{x} \text { is a } Y \text {-parameter sequence } & \Longleftrightarrow \operatorname{cmd}_{R} \mathrm{~K}(\boldsymbol{x} ; Y)=\operatorname{cmd}_{R} Y \\
& \Longleftrightarrow \operatorname{amp~} \mathrm{K}(\boldsymbol{x} ; Y)^{\dagger c}=\operatorname{amp} Y^{\dagger c} \\
& \Longleftrightarrow \operatorname{amp~} \mathrm{K}\left(\boldsymbol{x} ; Y^{\dagger c}\right)=\operatorname{amp} Y^{\dagger c} \\
& \Longleftrightarrow \boldsymbol{x} \text { is a } Y^{\dagger c} \text {-sequence. }
\end{aligned}
$$

The first biconditional follows by 3.5 (b) and the last by 3.5 (a). Since $K(\boldsymbol{x})$ is a bounded complex of free modules (hence of finite projective dimension), it follows from [3, Thm. (3.17)] that also $\mathrm{K}(\boldsymbol{x} ; Y)$ is $C$-reflexive, and the second biconditional is then immediate by the CMD-formula [3, Cor. (3.8)]. The third one is established as follows:

$$
\begin{aligned}
\mathrm{K}(\boldsymbol{x} ; Y)^{\dagger c} & \simeq \mathbf{R H o m}_{R}\left(\mathrm{~K}(\boldsymbol{x}) \otimes_{R}^{\mathbf{L}} Y, C\right) \\
& \simeq \mathbf{R H o m}_{R}\left(\mathrm{~K}(\boldsymbol{x}), Y^{\dagger c}\right) \\
& \simeq \mathbf{R H o m}_{R}\left(\mathrm{~K}(\boldsymbol{x}), R \otimes_{R}^{\mathbf{L}} Y^{\dagger c}\right) \\
& \simeq \mathbf{R} \operatorname{Hom}_{R}(\mathrm{~K}(\boldsymbol{x}), R) \otimes_{R}^{\mathbf{L}} Y^{\dagger c} \\
& \sim \mathrm{K}(\boldsymbol{x}) \otimes_{R}^{\mathbf{L}} Y^{\dagger c} \\
& \simeq \mathrm{K}\left(\boldsymbol{x} ; Y^{\dagger c}\right),
\end{aligned}
$$

where the second isomorphism is by adjointness and the fourth by, socalled, tensor-evaluation, cf. [1, (1.4.2)]. It is straightforward to check that $\operatorname{Hom}_{R}(\mathrm{~K}(\boldsymbol{x}), R)$ is isomorphic to the Koszul complex $\mathrm{K}(\boldsymbol{x})$ shifted $n$ degrees to the right, and the symbol $\sim$ denotes isomorphism up to shift.

If $C$ is a semi-dualizing complex for $R$, then both $C$ and $R$ are $C$-reflexive complexes, cf. [3, (2.8)], so we have an immediate corollary to the theorem:

Corollary 3.10. If $C$ is a Cohen-Macaulay semi-dualizing complex for $R$, then the following hold for a sequence $\boldsymbol{x}=x_{1}, \ldots, x_{n}$ in $\mathrm{m}$.

(a) $\boldsymbol{x}$ is a $C$-parameter sequence if and only if it is an $R$-sequence. 
(b) $\boldsymbol{x}$ is an $R$-parameter sequence if and only if it is a $C$-sequence.

ACKNOWLEDGEMENTS. The author would like to thank professor Srikanth Iyengar and professor Hans-Bjørn Foxby for taking the time to discuss the material presented here.

\section{REFERENCES}

1. Avramov, Luchezar L. and Foxby, Hans-Bjørn, Ring homomorphisms and finite Gorenstein dimension, Proc. London Math. Soc. (3) 75 (1997), 241-270.

2. Bruns, Winfried and Herzog, Jürgen, Cohen-Macaulay Rings, revised ed., Cambridge Stud. Adv. Math. 39 (1998).

3. Christensen, Lars Winther, Semi-dualizing complexes and their Auslander categories, Trans. Amer. Math. Soc. 353 (2001), 1839-1883.

4. Christensen, Lars Winther, Sequences for complexes, Math. Scand. 89 (2001), 1-20.

5. Foxby, Hans-Bjørn, Hyperhomological algebra \& commutative rings, notes in preparation.

6. Foxby, Hans-Bjørn, Bounded complexes of flat modules, J. Pure Appl. Algebra 15 (1979), $149-172$.

7. Hartshorne, Robin, Residues and Duality, Lecture Notes Math. 20 (1966).

8. Matsumura, Hideyuki, Commutative Ring Theory, second ed., Cambridge Stud. Adv. Math. 8 (1989).

CRYPTOMATHIC A/S

CHRISTIANS BRYGGE 28,2

DK-1559 COPENHAGEN V

DENMARK

E-mail: lars.winther@cryptomathic.com 